\title{
W Physics Result of PHENIX
}

\author{
Sanghwa PARK* for the PHENIX Collaboration \\ Seoul National University \\ e-mail: sanghwa@snu.ac.kr
}

\begin{abstract}
Measuring the flavor-separated quarks polarization is one of the primary goals in the PHENIX experiment at RHIC in order to understand the proton spin structure. The sea quark parton distriubution fuction (PDF) is not well known while the valence quark PDF is well constrained. The asymmetry of $\mathrm{W}$ boson production $A_{L}$ from polarized proton is sensitive to the helicity distribution of quarks and anti-quarks, so that the asymmetry measurement enables to access the sea quark contribution to the proton spin. RHIC provides us unique opportunity to perform this measurement using the longitudinally polarized proton collisions at center of mass energy up to $\sqrt{s}=$ $510 \mathrm{GeV}$. PHENIX has measured W boson through their leptonic decays into electrons at central rapidity $(|\eta|<0.35)$ and muons at forward/backward rapidities $(1.2<|\eta|<2.2)$. In succession to the first measurement performed in 2011, PHENIX collected twice of the data with integrated luminosity of $50 \mathrm{pb}^{-1}$ in 2012 with fully upgraded detectors and triggering system. We will present recent results of $\mathrm{W}$ to electron and muon decay channels and the prospect of the further analysis.
\end{abstract}

XXI International Workshop on Deep-Inelastic Scattering and Related Subject -DIS2013, 22-26 April 2013

Marseilles, France

\footnotetext{
*Speaker.
} 


\section{Introduction}

Understanding the structure of the proton spin has been a long-standing challenge. It is considered that the proton spin is composed of quarks, gluons and orbital angular momenta between quarks and gluons. Polarized Deep Inelastic Scattering (DIS) experiments provided measurements of the polarized parton distributions functions (PDF) of quarks, and total quark contribution to the proton spin is measured to be about 30\% [1]. Semi-Inclusive DIS (SIDIS) measurements can be used to constrain flavor-seprated polarized PDFs by measuring a hadron in the final state, but it is limited by large uncertaintyies of the fragmentation fuctions. $\mathrm{W}$ boson production in polarized $\mathrm{p}+\mathrm{p}$ collisions enables us to measure the quark and anti-quark contributions to the proton spin. As a manifestation of the maximum parity violation property of the weak interation, a $\mathrm{W}$ boson only couples to the left-handed quarks and right-handed anti-quarks. At RHIC energy of $\sqrt{s}=510 \mathrm{GeV}$, we can consider that $W^{+}$is produced by $\mathrm{u}$ and $\overline{\mathrm{d}}$ while $W^{-}$by $\overline{\mathrm{u}}$ and $\mathrm{d}$, and thus the flavor is almost fixed.

At RHIC, proton beams collide with alternate polarization signs, so that we can construct the parity violationg single spin asymmetry as:

$$
A_{L}^{W}=\frac{1}{P} \frac{N_{+}-N_{-}}{N_{+}+N_{-}}
$$

where $N_{+} / N_{-}$is the W yields for positive/negative proton beam helicity. PHENIX has measured the single spin asymmetries of $\mathrm{W}$ bosons through their leptonic decays, $W^{ \pm} \rightarrow e^{ \pm}$at midrapditiy[2] and $W^{ \pm} \rightarrow \mu^{ \pm}$at forward/backward rapidities. We have collected data with an integrated luminosity of $25 \mathrm{pb}^{-1}$ and $50 \mathrm{pb}^{-1}$ in 2011 and 2012, respectively. Moreover, a dedicated data taking for the $\mathrm{W}$ measurement has been performed in 2013, and we accumulated data with the total integrated luminosity of $156 \mathrm{pb}^{-1}$. Using the global ananlysis framework developed by the DSSV group and pseudo-experiment data of $200 \mathrm{pb}^{-1}$ at RHIC, it is expected to reduce the uncerstainty of PDFs significantly[3].

\section{PHENIX Experiment}

PHENIX has two central arm spectrometers and two forward muon arms. Figure 1 show schematic drawings of the PHENIX detectors. The two central arm spectrometers each covers the pseudo-rapidity range of $|\eta|<0.35$ and $\phi / 2$ in azimuth. The top panel of Fig. 1 shows a beam view of the central arm detectors. We use drift chambers (DC), pad chambers (PC), and electromagnetic calorimeters (EMCal) for the central arm measurement that observes electrons and positrons from $W^{-}$and $W^{+}$decays, respectively. At midrapidity, a $\mathrm{W}$ event can be idenfied by the Jacobian peak at $p_{T}$ of about half of $\mathrm{W}$ mass $(40 \mathrm{GeV} / \mathrm{c})$. Thus, we measure high- $p_{T}$ electrons/positrons using DC tracks and matching them to EMCal clusters. Most of the background is reduced by applying a relative isolation cut that requires the energy sum of a cluster divided by the energy of $\mathrm{e}^{ \pm}$candidates in a cone of $\mathrm{R}=0.4$ to be less than $10 \%$ of the candidates' energy.

The PHENIX muon arms cover a pseudo-rapidity range of $-2.2<\eta<-1.4$ in south and $1.4<\eta<2.4$ in north, and they have full azimuthal coverage. The detectors used for the $W^{ \pm} \rightarrow \mu^{ \pm}$ measurement are beam beam counters (BBC), muon tracking chambers (MuTr), muon identifiers 


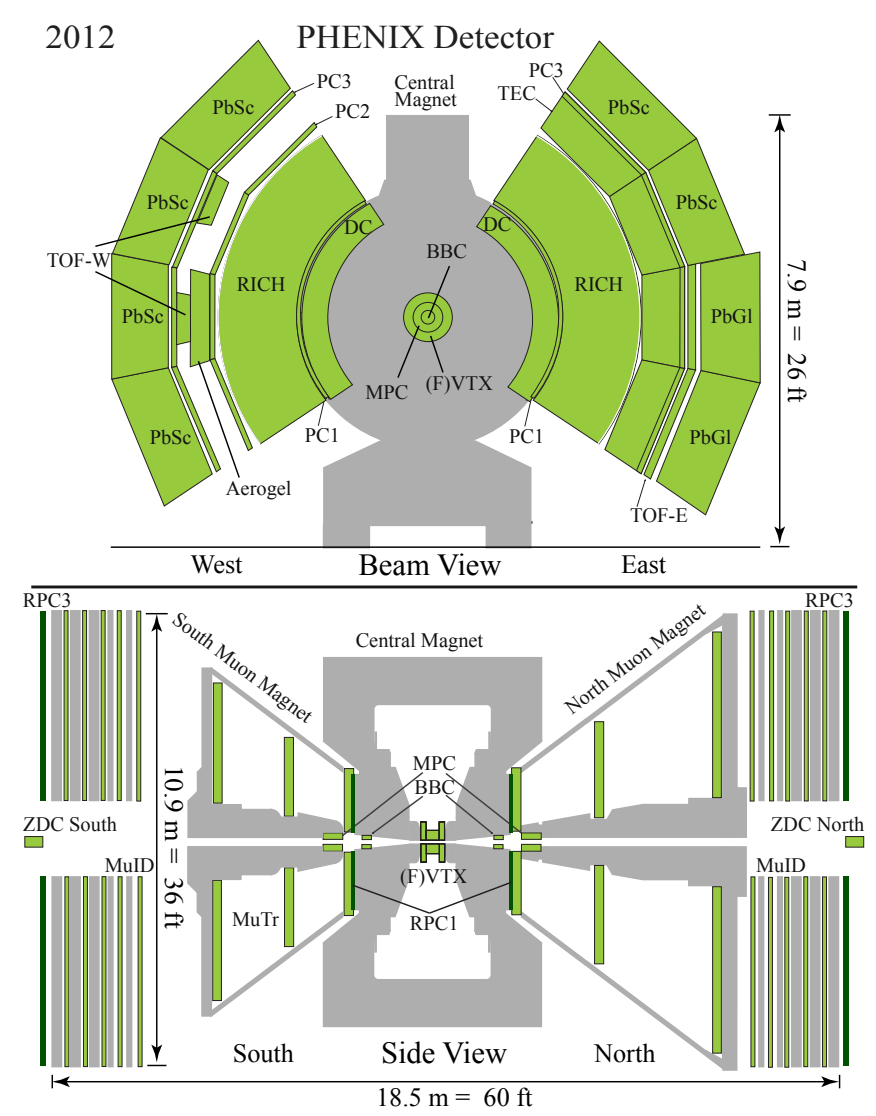

Figure 1: The schematic view of PHENIX detectors. The top panel shows the central arm, and the bottom panel shows a side view of PHENIX muon arm detectors.

(MuID), and resisitive plate chambers (RPC). The bottom panel in Fig. 1 shows the global and muon detectors. The muon arm consists of MuTr, that is a cathode strip chambers for tracking charged muon, followed by MuID that has a sandwich structure of five layers of Iarocci tubes and five iron absorbers. The single muon yields from $\mathrm{W}$ boson decays are dominant in the transverse momentum $p_{T}>15 \mathrm{GeV} / \mathrm{C}$ region compared to muon backgrounds which are mainly from open heavy flavor and quarkonia, while the momentum threshold of MuID is about $2.8 \mathrm{GeV} / \mathrm{c}$. To acheive sufficient trigger rejection for $\mathrm{W}$-detection, we introduced fast readout electronics in addition to the exisiting MuTr front-end electronics. Besides, the RPCs were installed in 2011 for the better trigger performance based on its good timing resoultion and muon identification. One of RPCs is located in front of the first chamber of MuTr, and the other is behind of MuID. High momentum tracks are triggered using their small bending at the MuTr in radial magnetic field and timing match using BBC and RPC.

Unlike the central arm measurement, the Jacobian peak is kinematically suppressed at forward/backward rapidities, and that is not a good discriminant to distinguish signal from background, so the background estimation is a challenging issue in the forward arm analysis. Therefore, we introduced a likelihood approach to reduce background and to estimate the signal to background 

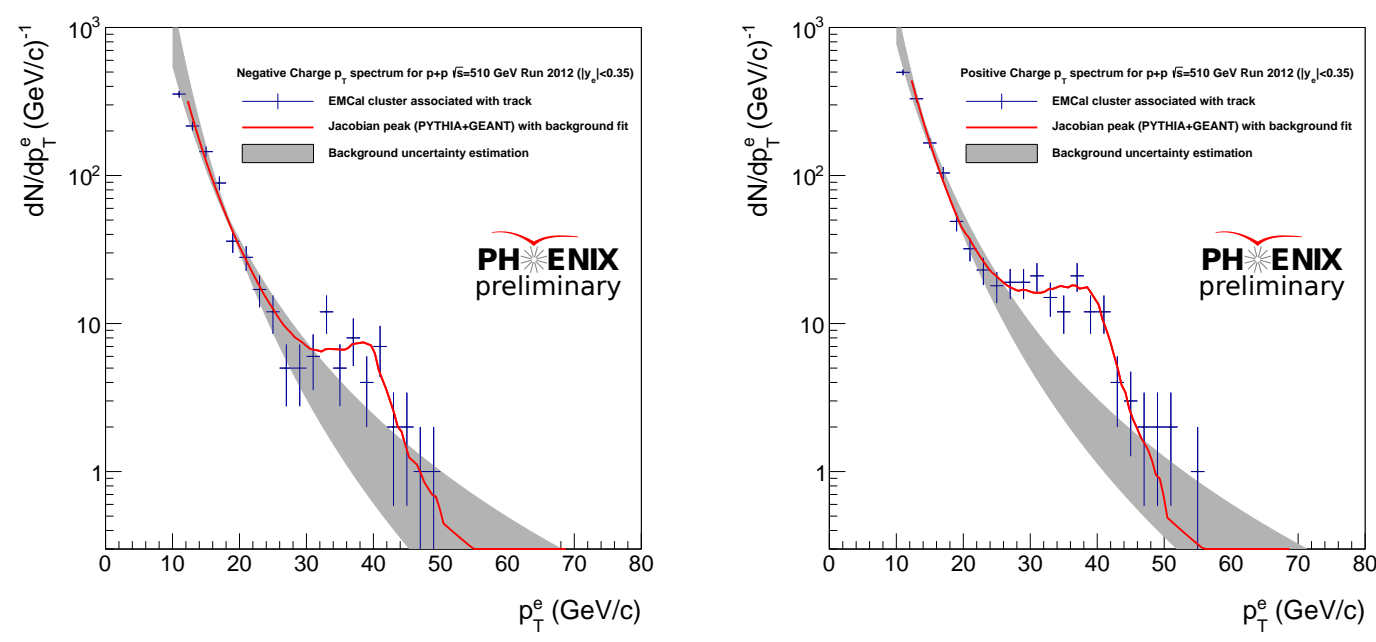

Figure 2: $P_{T}$ spectrum for electrons(left) and positrons(right) measured in midrapidity region.

ratio. First, we used multivariate cut for pre-selection of signal by composing likelihood $\lambda$ for signal and background and selecting the higher region of the ratio between $\lambda_{\text {sig }}$ and $\left(\lambda_{\text {sig }}+\lambda_{B G}\right)$. The signal to background ratio after the pre-selection is estimated by an extended unbinned maximum likelihood fitting using two kinematic variable distributions, pseudo-rapidity and azimuthal bending between the second and third MuTr chambers normalized by the transverse momentum and radial angle of track. The discrepancy between true momentum and reconstructed momentum is significant in case of background than true high momentum tracks. Using those variables, we extracted the signal and muon background shapes from simulation, while hadronic background from data-driven way, and then performed the fitting to data to estimate the background fraction.

\section{Result}

Figure 2 shows the $p_{T}$ specturm of $W^{ \pm} / \mathrm{Z}$ at midrapidity with background uncertainty estimation band and spectrum fitting result. Background is dominant at $10<p_{T}<30 \mathrm{GeV} / \mathrm{c}$, and the signal region is 30 to $50 \mathrm{GeV} / \mathrm{c}$. We performed a power law fitting for background in $10<p_{T}<70$ $\mathrm{GeV} / \mathrm{c}$, and a power law and jacobian peak fitting for $20<p_{T}<50 \mathrm{GeV} / \mathrm{c}$ to estimate the remaining background fraction. In Figure 2, we can see that the signal peaks at around $40 \mathrm{GeV} / \mathrm{c}$ as expected.

Figure 3 shows PHENIX preliminary $A_{L}^{W}$ results along with theoritical predictions. The top panel shows $A_{L}$ for $W^{+}+Z$ to $\mu^{+}$at forward/backward rapidities and $\mathrm{e}^{+}$at central rapidity. The result for $W^{-}$is shown in the bottom panel of Fig. 3. Since two decay modes use different energy cuts, theory curves are shown as dashed lines for midrapidity measurement, and as solid lines for forward/backward measurement. The measured $A_{L}^{W}$ result is consistent with theoritical calculations within the uncertainty range. For the preliminary result of $A_{L}^{W}$ at forward/backward rapidities, the systemtic uncertainty is considered by taking a conservative factor of 2 in the signal and background ratio. 

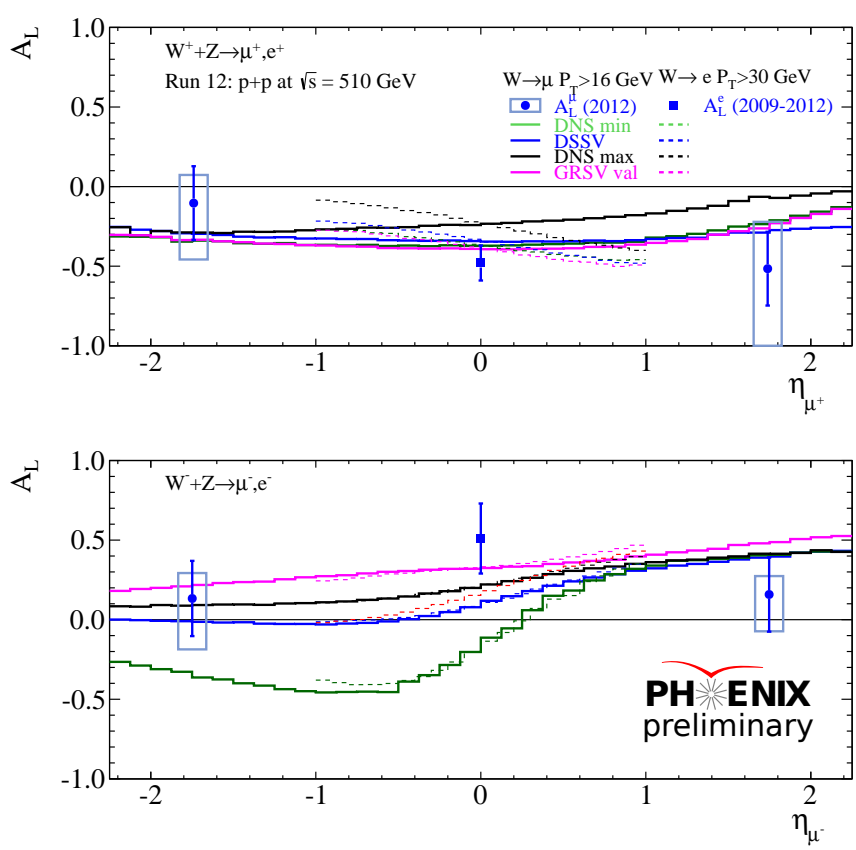

Figure 3: $A_{L}$ for $W^{+}$(top) and $W^{-}$(bottom) at central and forward/backward rapidities. Various theoritical predictions are shown together as dashed line for midrapidity and solid lines for forward/backward rapidities.

\section{Conclusion and outlook}

PHENIX accumulated data of $50 \mathrm{pb}^{-1}$ in 2011 and 2012, and the preliminary results are presented. The measured values of the single spin asymmetries were consistent with theory models within the uncertainly ranges. Further analysis is in progress to finalize and improve these results through additional detectors and increased statistics. For $W^{ \pm} \rightarrow \mu^{ \pm}$decay channel, a forward silicon vertex (FVTX) was installed and operated successfully in 2012, and it is expected to give us further background rejection. In 2013, we have completed high statistics data taking with an integrated luminosity of $156 \mathrm{pb}^{-1}$, and it will enable us to constrain anti-quark PDFs significantly.

\section{References}

[1] Ashman J, et al (European Muon Collaboration), Phys. Lett. B 206, 364 (1988).

[2] A. Adare et al. (PHENIX Collaboration), Phys. Rev. Lett. 106, 062001 (2011).

[3] D.de Florian and W Vogelsang, Phys.Rev.D 81 (2010), 094020. 0.1, 2. 